\title{
AN EXTENSION OF DEDEKIND'S LINEAR INDEPENDENCE THEOREM
}

\author{
CHARLES M. WALTERS ${ }^{1}$
}

Abstract. Dedekind's theorem on the linear independence of isomorphisms of a field is extended to the case of linear independence of compositions of isomorphisms and powers of a derivation, $D$, for fields of characteristic zero which contain an element $s$ such that $D(s)=1$.

In this paper we present an extension of the following classical theorem on the linear independence of isomorphisms of a field [1, p. 25].

TheOREM 1 [Dedekind]. Let $E$ and $P$ be fields. Then the collection $\{F \mid F$ is an isomorphism of $E$ into $P\}$ is left linearly independent over $P$; i.e., $\sum_{i=1}^{n} p_{i} F_{i}=0, p_{i}$ in $P, n \geqq 1$ an integer, and $F_{i} \neq F_{j}$ for $i \neq j$ implies $p_{i}=0$ for $i=1,2, \cdots, n$.

For fields of characteristic zero, the above theorem may be extended to the case of compositions of isomorphisms and powers of a derivation on $E$ as in the following theorem. This result is useful in the study of linear transformations on the field of Mikusiński operators [2].

THEOREM 2. Let $E$ and $P$ be fields of characteristic zero, $D$ a derivation of $E$ into $E$, and $s$ an element in $E$ such that $D(s)=1$. Then, the collection $\left\{F D^{n} \mid F\right.$ is an isomorphism of $E$ into $P$ and $n \geqq 0$ is an integer $\}$ is left linearly independent over $P$.

In the following we consider elements of a field $E$ as endomorphisms of $E$ defined for $b$ in $E$ by $b(x)=b x$ for each $x$ in $E$. (A field $E$ can be embedded in the ring of group endomorphisms on the additive group $E$.) For the proof of Theorem 2, we will need a lemma.

Lemma. Let $E$ and $P$ be fields of characteristic zero, $D$ a derivation of $E$ into $E$, and $s$ an element in $E$ such that $D(s)=1$. If $F$ and $G$ are homomorphisms of $E$ into $P$ such that $F D^{p}=G D^{q}$ for some integers $p, q \geqq 0$, then $p=q$ and $F=G$.

Received by the editors August 28, 1972.

AMS (MOS) subject classifications (1970). Primary 13B10.

Key words and phrases. Derivation, linearly independent.

1 These results are contained in the author's doctoral dissertation written with the guidance of Professor Raimond A. Struble at North Carolina State University, Raleigh.

(c) American Mathematical Society 1973 
Proof. If $p=q=0$, the result is trivial. It is easily verified that for integers $p, q \geqq 0$

$$
\begin{aligned}
D^{p}\left(s^{q}\right) & =0 & & \text { if } p>q, \\
& =q(q-1) \cdots(q-p+1) s^{q-p} & & \text { if } p \leqq q .
\end{aligned}
$$

Hence if $p>q \geqq 0$, then $0=F D^{p}\left(s^{q}\right)=G D^{q}\left(s^{q}\right)=q$ ! is a contradiction and by the symmetry of this argument we conclude that $p=q$. Now suppose $m>0$ is the smallest integer such that $F D^{m}=G D^{m}$. Then, if $x$ is an element of $E$, from Leibnitz' formula

it follows that

$$
D^{m}(s x)=\sum_{r=0}^{m}\left(\begin{array}{l}
m \\
r
\end{array}\right) D^{r}(s) D^{m-r}(x)
$$

$$
D^{m} s=\sum_{r=0}^{m}\left(\begin{array}{c}
m \\
r
\end{array}\right) D^{r}(s) D^{m-r} .
$$

But $D^{0}(s)=s, D(s)=1$, and $D^{r}(s)=0$ for $r \geqq 2$. Thus, (1) reduces to

$$
D^{m} s=s D^{m}+m D^{m-1} .
$$

Since $F$ is multiplicative, if $x$ is in $E$ then $F s(x)=F(s x)=F(s) F(x)$ and so $F s=F(s) F$. Therefore, from (2) we obtain

so that

$$
\begin{aligned}
F D^{m} s & =F\left(s D^{m}+m D^{m-1}\right)=F(s) F D^{m}+m F D^{m-1} \\
& =G(s) G D^{m}+m G D^{m-1}=G D^{m} s
\end{aligned}
$$

$$
[F(s)-G(s)] F D^{m}=m\left[G D^{m-1}-F D^{m-1}\right] .
$$

Moreover, $F D^{m}\left(s^{m+1}\right)=G D^{m}\left(s^{m+1}\right)$ implies $F(s)=G(s)$ and so from the above we have $G D^{m-1}=F D^{m-1}$, a contradiction of the choice of $m$.

Proof OF Theorem 2. Suppose there exists a set of elements $b_{1}, b_{2}, \cdots$, $b_{m}$ in $P$, not all zero, and a set of $m$ distinct mappings $F_{1} D^{n_{1}}, F_{2} D^{n_{2}}, \cdots$, $F_{m} D^{n_{m}}$ such that

$$
\sum_{i=1}^{m} b_{i} F_{i} D^{n_{i}}=0
$$

where we assume without loss of generality that $n_{1} \geqq n_{2} \geqq \cdots \geqq n_{m} \geqq 0$. Then there exists such a relation exhibiting a smallest value $n_{1}$; i.e., exhibiting a minimum largest power of $D$, say

$$
\sum_{i=1}^{p} b_{i} F_{i} D^{n_{i}}=0
$$

with $F_{1} D^{n_{1}}, F_{2} D^{n_{2}}, \cdots, F_{p} D^{n_{p}}$ distinct, $b_{1}, b_{2}, \cdots, b_{p}$ not all zero, and $n_{1} \geqq n_{2} \geqq \cdots \geqq n_{p} \geqq 0$. Now if $n_{1}=0$, then Theorem 1 applies. Hence we 
may assume that $n_{1}>0$ If $p=1$, then $b_{1} F_{1} D^{n_{1}}(x)=0$ for all $x$ in $E$ means $F_{1} D^{n_{1}}=0$. But $F_{1} D^{n_{1}} \neq 0$ since if so $F_{1}=0$ by the lemma. Thus we may assume that $p>1$. We rewrite the relation (3) as

$$
\sum_{i=1}^{l} b_{i} F_{i} D^{n_{1}}+\sum_{i=l+1}^{p} b_{i} F_{i} D^{n_{i}}=0
$$

where $n_{i}<n_{1}$ for $l+1 \leqq i \leqq p$. Now if (4) holds then we may choose such a relation having a minimum $l$; i.e., having a minimum number of terms involving the maximum power $n_{1}$ of $D$. Thus we assume that in (4) $l$ is minimum. Multiplying (4) on the right by $s$, in view of (2) and the fact that each $F_{i}$ is multiplicative we obtain

$$
\sum_{i=1}^{l} b_{i} F_{i}(s) F_{i} D^{n_{1}}+\sum_{i=1}^{l} n_{1} b_{i} F_{i} D^{n_{1}-1}
$$

$$
+\sum_{i=l+1}^{p} b_{i} F_{i}(s) F_{i} D^{n_{i}}+\sum_{i=l+1}^{p} n_{i} b_{i} F_{i} D^{n_{i}-1}=0 .
$$

Multiplying (4) on the left by $F_{1}(s)$ and subtracting the result from (5) yields

$$
\sum_{i=2}^{l} b_{i}\left[F_{i}(s)-F_{1}(s)\right] F_{i} D^{n_{1}}+\sum_{i=1}^{l} n_{1} b_{i} F_{i} D^{n_{1}-1}
$$

$$
+\sum_{i=l+1}^{p} b_{i}\left[F_{i}(s)-F_{1}(s)\right] F_{i} D^{n_{i}}+\sum_{i=l+1}^{p} n_{i} b_{i} F_{i} D^{n_{i}-1}=0 .
$$

Suppose $l=1$ in (4). Then (6) reduces to

$$
n_{1} b_{1} F_{1} D^{n_{1}-1}+\sum_{i=2}^{p} b_{i}\left[F_{i}(s)-F_{1}(s)\right] F_{i} D^{n_{i}}+\sum_{i=2}^{p} n_{i} b_{i} F_{i} D^{n_{i}-1}=0 .
$$

Now in (7) no term in the last sum corresponds to $F_{1} D^{n_{1}-1}$ since if $F_{1} D^{n_{1}-1}=F_{i} D^{n_{i}-1}$ for some $i \geqq 2$, then $F_{1} D^{n_{1}}=F_{i} D^{n_{i}}$ which contradicts the assumption that the $F_{j} D^{n_{j}}$ are distinct. Moreover if $F_{1} D^{n_{1}-1}$ appears in a term in the first sum in (7), say $F_{1} D^{n_{1}-1}=F_{i} D^{n_{i}}$ for some $i \geqq 2$, then its coefficient is zero since $F_{1}=F_{i}$ by the lemma. Therefore $F_{1} D^{n_{1}-1}$ appears in (7) with the nonzero coefficient $n_{1} b_{1}$. Hence if $l=1$, (7) contradicts the choice of $n_{1}$. Suppose $l>1$ in (4). Then, since $n_{1}>n_{1}-1 \geqq n_{l+1} \geqq$ $n_{l+2} \geqq \cdots \geqq n_{p} \geqq 0$, in (6) only the first sum contains terms in which $D$ appears to the power $n_{1}$. Thus if $F_{i}(s) \neq F_{1}(s)$ for some $2 \leqq i \leqq l$, then (6) contradicts the choice of $l$. If, on the other hand, $F_{i}(s)=F_{1}(s)$ for each $2 \leqq i \leqq l$ then as before (6) contradicts the choice of $n_{1}$ since the first sum 
vanishes and $F_{1} D^{n_{1}-1}$ appears with the nonzero coefficient $n_{1} b_{1}$. Therefore we conclude that no minimum $l$ exists and so the relation (3) holds only if $b_{i}=0$ for $i=1,2, \cdots, p$ which concludes the proof.

Corollary. Let $E$ be a field of characteristic zero, $D$ a derivation of $E$ into $E$, and $s$ an element in $E$ such that $D(s)=1$. Then the collection $\left\{D^{n} \mid n \geqq 0\right.$ is an integer $\}$ is left linearly independent over $E$.

AcKnowledgement. The author is grateful to Professor Jiang Luh for his valuable comments.

\section{REFERENCES}

1. N. Jacobson, Lectures in abstract algebra. Vol. III: Theory of fields and Galois theory, Van Nostrand, Princeton, N.J., 1964. MR 30 \#3087.

2. C. M. Walters, Continuous linear transformations on the field of Mikusiński operators, Ph.D. Thesis, North Carolina State University, Raleigh, N.C., 1971.

Air Force Weapons laboratory, Kirtland Air Force Base, New Mexico 87117

Current address: Research and Analysis Division, Directorate of Personnel Planning, Headquarters USAF, Washington, D.C. 20330 